\title{
Impact of cross sections uncertainties on the study of galactic cosmic-ray propagation with the DRAGON2 code
}

\author{
de la Torre Luque, P., ${ }^{a, *}$ M. N. Mazziotta, ${ }^{b}$ F. Loparco, ${ }^{b, c}$ F. Gargano ${ }^{b}$ and D. Serini ${ }^{b, c}$ \\ ${ }^{a}$ The Oskar Klein Centre, Department of Physics, Stockholm University, AlbaNova \\ SE-10691 Stockholm, Sweden \\ ${ }^{b}$ Istituto Nazionale di Fisica Nucleare, Sezione di Bari, via Orabona 4, I-70126 Bari, Italy \\ ${ }^{c}$ Dipartimento di Fisica "M. Merlin" dell'Università e del Politecnico di Bari, via Amendola 173, I-70126 \\ Bari, Italy \\ E-mail: pedro.delatorreluque@fysik.su.se
}

The study of galactic cosmic rays provides a valuable tool for understanding better the galactic environment, from the evolution of turbulence in the interstellar plasma to the exploration of new physical phenomena. Modern cosmic-ray experiments have reached very high precision in a wide energy range, which allow us to perform detailed tests on out models of particle propagation in the Galaxy. Nevertheless, these predictions are limited to other key piece: spallation cross sections measurements. The lack of data at energies above a few $\mathrm{GeV} / \mathrm{n}$, where most of the CR data are available, and the huge amount of interaction channels for production of different isotopes involved in the so-call spallation network make our predictions to be very uncertain (in some cases at the level of 50\%). Therefore, in this talk we explore the consequences of cross sections uncertainties in the evaluation of the spectra of secondary CRs and the predictions on the propagation parameters that govern their production in the Galaxy. We quantify these uncertainties and examine their impact on our conclusions about the nature of secondary CRs and their propagation. In addition, due to the importance of spallation cross sections measurements in our codes of CR propagation, we show new ways to refine current cross sections parametrizations by combining different CR observables and reduce the impact of cross sections uncertainties on our predictions.

$37^{\text {th }}$ International Cosmic Ray Conference (ICRC 2021)

July 12th - 23rd, 2021

Online - Berlin, Germany

\footnotetext{
${ }^{*}$ Presenter
} 


\section{Introduction}

After cosmic rays (CRs) are injected by galactic sources (mainly supernova remnants), these particles continuously undergo collision-less interactions with plasma waves generated in the interstellar medium (ISM), making their transport to be characterized by a diffusive motion which is not restricted to the galactic plane, but that extends above and below the disk several kiloparsecs [1]. The amount of secondary CRs (i.e. those generated from spallation reactions of primary CRs with gas nuclei in the ISM) provides crucial information about the galactic environment and the propagation process, allowing us to study in detail their diffusive motion.

In particular, the flux ratio of a secondary CR species to a primary CR species is very sensitive to the propagation parameters [2], and mostly insensitive to the source spectrum of primary species, which offers a valuable tool to study the interactions of CRs during their propagation. The flux ratio between a secondary $\mathrm{CR}$ species to a primary $\mathrm{CR}$ species can be approximately written as:

$$
\frac{J_{k}}{J_{i}}(R) \approx \frac{\tau_{d i f f}(E) n_{I S M} \sigma_{i \rightarrow k} J_{i}(R)}{J_{i}(R)} \sim \sigma_{i \rightarrow k}(R) \tau_{\text {diff } f}(R) \propto \sigma_{i \rightarrow k}(R) / D(R) .
$$

Here, $R$ stands for the rigidity of CR particles, $\sigma_{i \rightarrow k}$ is the production cross section of production of the secondary CR species $k$ from the interaction of the primary CR species $i$ with the gas in the ISM (spallation reactions), $J$ is the flux of these particles and $n_{I S M}$ is the density of gas in the ISM. Finally, $\tau_{\text {diff } f} \propto 1 / D(R)$ is the diffusion time of CRs in the Galaxy, where $D(R)$ is the diffusion coefficient used to describe the propagation of CRs in the Galaxy.

With the increasing precision of the measurements of CR fluxes, we are now able to precisely evaluate our models of CR propagation in a large energy range. Nevertheless, there are important uncertainties ( $>20 \%$ ) coming from the measurements of cross sections of production of the secondary CRs which are interesting for these kind of studies (mainly B, Be and Li) [3-5]. For most of the interaction channels there are hardly no cross sections measurements above a few $\mathrm{GeV} / \mathrm{n}$, while for some other channels there is no experimental data at all. This imposes serious difficulties in the study of the nature of secondary CRs and the propagation process. In order to reduce the impact of the lack of data, we implement in our propagation codes parametrizations and extrapolations of the spallation cross sections involved in the CR network.

This work aims at showing the impact of these uncertainties in our predictions on the spectra of secondary CRs and how this can affect our conclusions about the transport of Galactic CRs and the origin of secondary CRs. To do so, we have studied different cross sections parametrizations and the cross sections experimental data in refs. [4, 5]. Here, we make use of a well tested customized version $^{1}[6]$ of the DRAGON2 CR propagation code [7, 8]. We solve the propagation equation assuming a 2-D model of the Galaxy structure and adopt a diffusion-reacceleration scheme. The diffusion coefficient used here can be generally written as:

$$
D(R)=D_{0} \beta^{\eta} \frac{\left(R / R_{0}\right)^{\delta}}{\left[1+\left(R / R_{b}\right)^{\Delta \delta / s}\right]^{s}},
$$

where $R_{0}=4 \mathrm{GV}$ is the reference rigidity, $R_{b}=312 \mathrm{GV}$ is the rigidity break, $\Delta \delta$ is the change in spectral index and $s=0.04$ is a smoothing parameter.

\footnotetext{
${ }^{1}$ This version is public at https://github.com/tospines/Customised-DRAGON2_beta/
} 


\section{Uncertainties associated to current cross sections measurements}

The basic structure of the cross sections parametrizations is based on a flat energy dependence above a few $\mathrm{GeV} / \mathrm{n}$ and a smoothly increasing energy dependence below. The region around a few hundreds $\mathrm{MeV} / \mathrm{n}$ is governed, in many interaction channels, by resonance peaks. Therefore, above a few $\mathrm{GeV} / \mathrm{n}$, where most of the CR flux measurements are found, the largest uncertainties in the estimation of the secondary CRs spectra come from the normalization of the parametrizations [9].

In figure 1, the $\mathrm{CR}$ flux ratios between $\mathrm{B}, \mathrm{Be}$ and $\mathrm{Li}$ predicted from three popular parametrizations, namely DRAGON2, GALPROP and Webber, are shown for different halo height values, to appreciate how this magnitude can change our predictions (see Ref. [4] for details), and are compared to AMS-02 data [10]. These ratios are extremely useful to compare different cross sections, since, above $\sim 10 \mathrm{GeV} / \mathrm{n}$, they mainly depend on their cross sections ratios. Remarkably, the energy dependence of these ratios above a few $\mathrm{GeV} / \mathrm{n}$ is quite similar for the different cross sections.

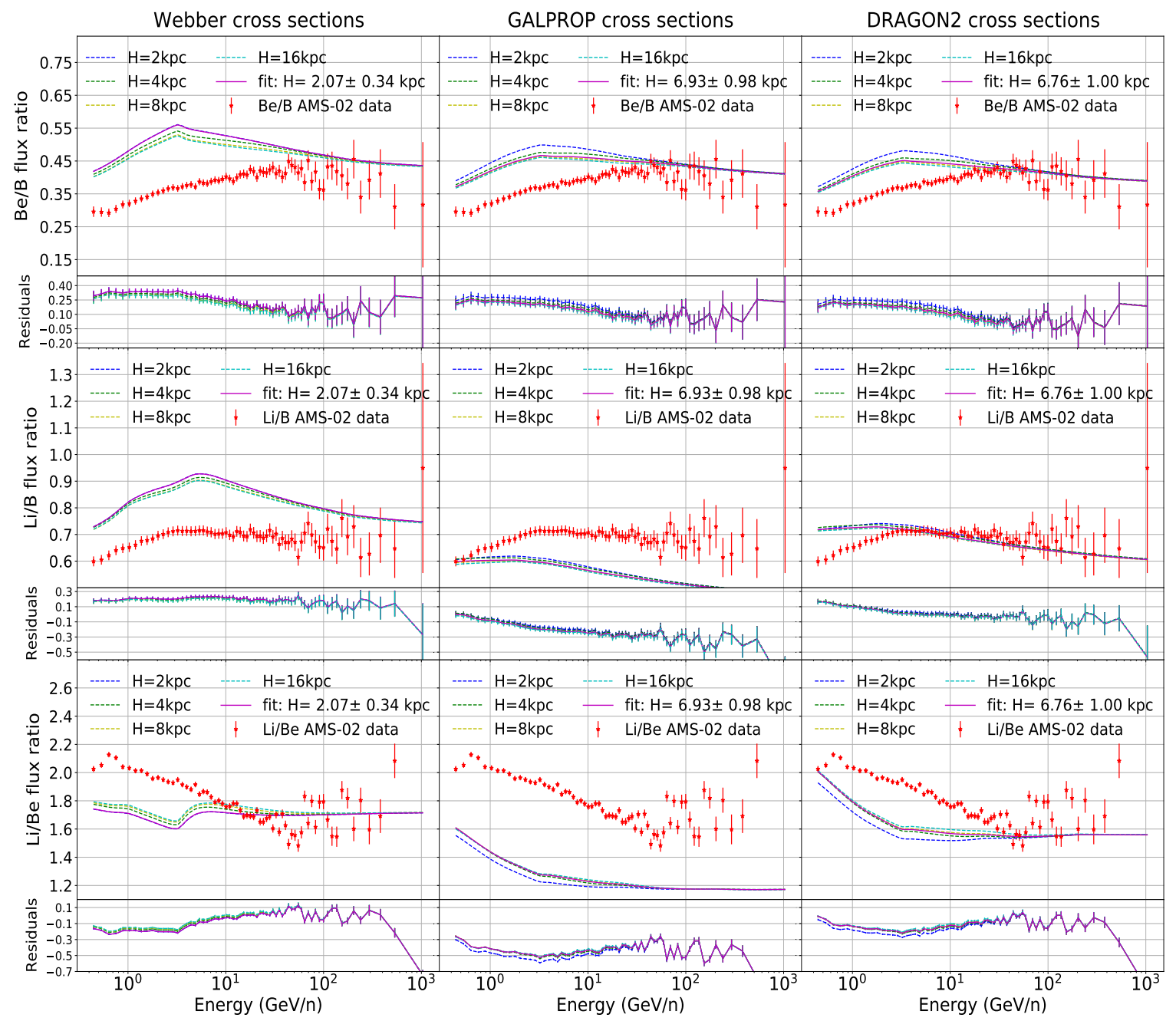

Figure 1: CR flux ratios among B, Be and Li for the cross sections parametrizations discussed in this work. These plots show the simulated spectra for various halo sizes, as well as for the halo size that provides the best fit to the flux ratios of ${ }^{10} \mathrm{Be}$. The diffusion coefficient is such that reproduces $\mathrm{B} / \mathrm{C}$ ratio in every case. 
Nevertheless, although the predicted energy dependence from these cross sections is similar, the predictions show important differences in their magnitude. Specially interesting is the underproduction (with respect to AMS-02 data) of the Li ratios predicted from the GALPROP cross sections, since this has lead to some researchers to propose an astrophysical source producing Li [11] (i.e. production of primary Li). Nevertheless, this does not happen with other cross sections' predictions and, as we show below, this can be more plausibly explained, considering cross sections uncertainties, which are specially large for $\mathrm{Li}$, given the lack of cross sections data on its production.

\subsection{Estimation of secondary CRs fluxes and the possible contributions from primary sources}

To show how the uncertainties in cross sections measurements affect the determination of the normalization of their parametrizations, we have derived two bracketing models that encompass the average $1 \sigma$ error in the measurements for each interaction channel of production of every isotope of $\mathrm{B}, \mathrm{Be}$ and $\mathrm{Li}$, from the primary $\mathrm{CRs}{ }^{12} \mathrm{C}$ and ${ }^{16} \mathrm{O}$. Then, we propagate these uncertainties to the secondary-to-secondary ratios predicted from the GALPROP parametrizations, demonstrating that the AMS-02 data lie between these two models. This is shown in figure 2 (panels a, b and c), where the yellow bands represent the values of the flux ratios within the two limiting models (including only the uncertainties related to the channels from ${ }^{12} \mathrm{C}$ and ${ }^{16} \mathrm{O}$, which are the best know channels). The expected full uncertainty bands on the flux ratios are also represented by black dashed lines.

Furthermore, we scaled the normalization of the parametrizations in order to simultaneously fit these secondary-to-secondary ratios above $20 \mathrm{GeV} / \mathrm{n}$, considering the adjustment which implies the minimum rescaling from the original parametrization. This fit yielded a renormalization of the cross sections of $\sim 5 \%$ down for the B flux, $\sim 18 \%$ down for the Be flux $\left(\sim 16 \%\right.$ in the ${ }^{12} \mathrm{C}$ channels and $\sim 20 \%$ in the ${ }^{16} \mathrm{O}$ channels) and of $\sim 28 \%$ up for the Li flux ( $\sim 22 \%$ for ${ }^{12} \mathrm{C}$ channels and $\sim 34 \%$ ${ }^{16} \mathrm{O}$ channels). We highlight that these factors can be affected by the gas density distribution used (we have observed variations of up to $\pm 4 \%$ for other gas density distributions), the inelastic cross sections employed (different realizations with different inelastic cross sections parametrizations lead to $<3 \%$ variations in these factors) and also multi-step reactions can affect this (finding up to $\sim 3 \%$ variations for different configurations).

On the other hand, recently the AMS-02 collaboration released the flux measurements on the next secondary CR species, Fluorine [12]. Again here, some authors claimed the necessity of adding a primary source of fluorine to explain its spectrum[13]. In panel $\mathrm{d}$ of figure 2 we show the fluorine spectrum predicted from the DRAGON2 cross sections parametrizations using the diffusion coefficient that fits the B/C spectrum reported by AMS-02. We highlight here the importance of reproducing the primary spectra of the $\mathrm{CRs} \mathrm{Ne}, \mathrm{Mg}$ and $\mathrm{Si}$ [14], since they are the main producers of fluorine (in particular, the stable isotope of $\mathrm{F}$ is ${ }^{19} F$ ). As we can see from the flat residuals, the energy dependence of the predicted fluorine spectrum is in perfect agreement with AMS-02, but there is an offset of around a $20 \%$, well inside the uncertainties related to the cross sections of $\mathrm{F}$ production, which can be as large as $50 \%$. Again here, we conclude that this discrepancy can be plausibly explained considering a $20 \%$ scale of the cross sections parametrizations.

We remark that the fact that we reproduce the energy dependence of the fluorine experimental data is noticeable since this CR species is formed mainly from $\mathrm{Ne}, \mathrm{Mg}$ and $\mathrm{Si}$, which are supposed to be injected from a different population (or, at least, distribution) of CR sources. 
a)
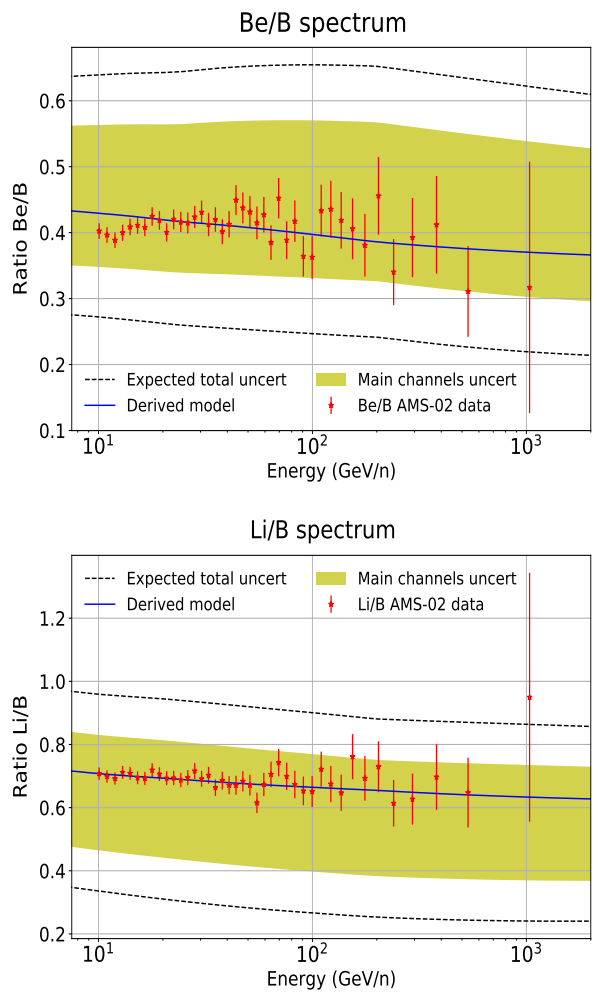

b)
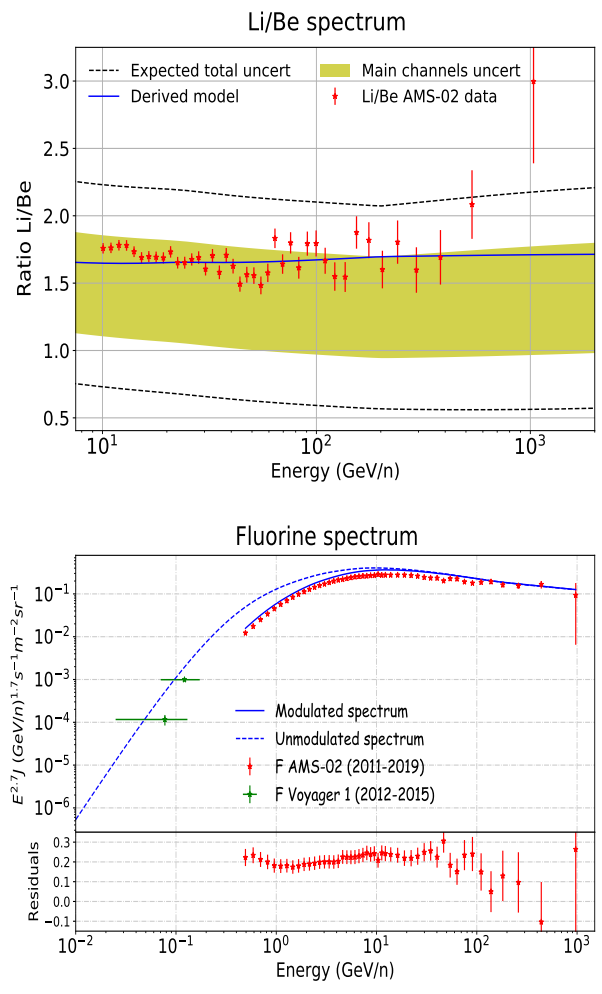

Figure 2: Panels a, b and c: Secondary-over-secondary flux ratios involving Li, Be and B. The yellow bands correspond to the uncertainties obtained using the bracketing cross section models for the main production channels. The bands between the black dashed lines correspond to the expected total uncertainties, obtained adding the contributions from all the minor production channels. The blue lines are obtained by simultaneously fitting the AMS-02 data. Panel d: Spectrum of fluorine predicted from the DRAGON2 parametrizations with the diffusion coefficient that reproduces the B/C spectrum reported by AMS-02.

\subsection{Impact on halo height estimations}

To show the effect of cross sections uncertainties in the estimation of the halo height, we have evaluated the ${ }^{10} \mathrm{Be} /{ }^{9} \mathrm{Be}$ flux ratios for various halo heights and determined the value that provides the best fit to experimental data (see Ref. [4] for all the details) for the DRAGON2, GALPROP and Webber parametrizations and the rescaled cross sections explained above, setting the diffusion coefficient to fit the $\mathrm{B} / \mathrm{C}$ ratio in each case. Figure 3, a, shows the halo height value that provides the best fit for each cross section model, the uncertainties coming from its determination ( $1 \sigma$ statistical uncertainties related to the fit) and a line indicating the mean value among these models, which is $5.6_{-0.85}^{+0.86} \mathrm{kpc}$, including the value determined from the Webber parametrizations, and the value obtained without this prediction is $6.8 \pm 1 \mathrm{kpc}$. Nevertheless, the $\sim \pm 1 \mathrm{kpc}$ error only represents the $1 \sigma$ uncertainty assuming no uncertainties in the evaluation of our model. Figure $3, \mathrm{~b}$, shows the ${ }^{10} \mathrm{Be} /{ }^{9} \mathrm{Be}$ flux ratio predicted with the DRAGON2 parametrizations, including the uncertainty band around the best-fit halo size value associated to the uncertainties in the normalization of the cross sections for the production of these isotopes from the channels of ${ }^{12} \mathrm{C}$ and ${ }^{16} \mathrm{O}$. From this band, we see that we are only able to constrain the halo height to be larger than $\sim 3 \mathrm{kpc}$. 


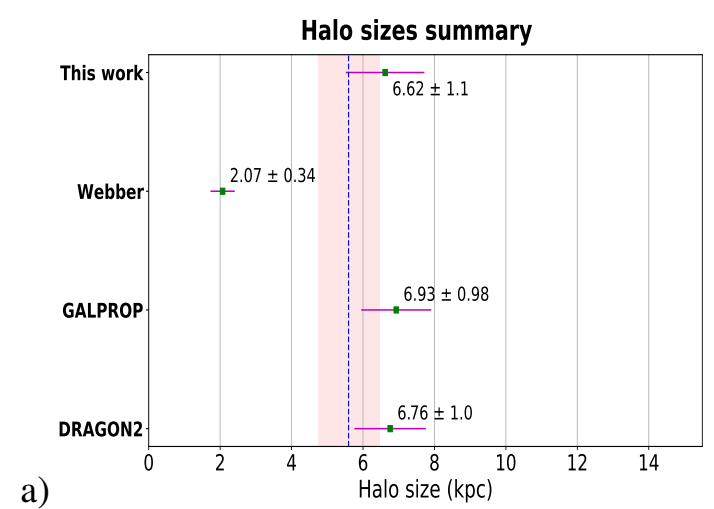

b)

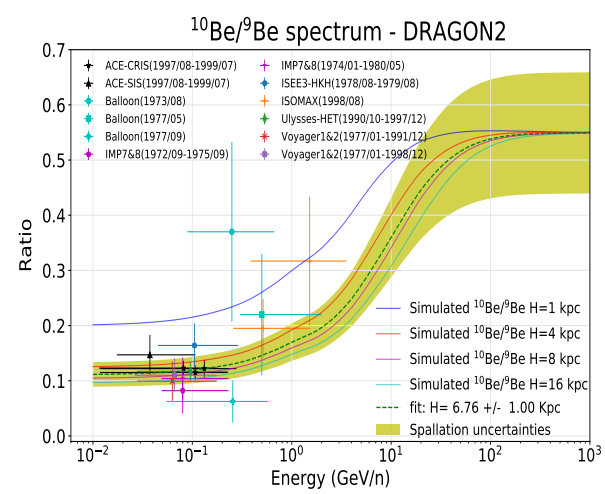

Figure 3: Panel a: Summary of the results obtained for the fits of halo sizes to the ${ }^{10} \mathrm{Be} /{ }^{9} \mathrm{Be}$ experimental data. Also the values obtained with the cross sections explained above are shown. Error bars reflect only statistical uncertainties, assuming no uncertainty in our predictions. The dashed blue line represents the mean value of the halo best fit values and the red band the uncertainties of the mean $(1 \sigma)$, calculated as the mean of the halo best fit values $\pm \sigma$. Panel b: ${ }^{10} \mathrm{Be} /{ }^{9} \mathrm{Be}$ predicted flux ratios for various halo height values compared to experimental data for the DRAGON2 cross section. The yellow band represents the error associated to the cross sections of production of these isotopes, as obtained from the procedure explained above.

\subsection{Diffusion parameters inferred from current cross sections parametrizations}

In this section, we show how different cross sections parametrizations and the different secondary CRs considered lead to slightly different predictions of the main propagation parameters. Full details can be found in Ref. [5]. We make use of a Markov-Chain Monte Carlo algorithm able to evaluate the probability distribution function for the experimental data (AMS-02 data [10]) to be described by a propagation model adjusted by a set of diffusion parameters $\left(D_{0}, \eta\right.$ and $\delta$, from eq. 2 , in addition to the effective alfvèn velocity, $V_{A}$, which determines the level of reacceleration that CRs experience) and their confidence intervals. The value of $\Delta \delta$ in eq. 2 is fixed here to 0.14 . Concretely, we focus on the the spectra of $\mathrm{B}, \mathrm{Be}$ and $\mathrm{Li}$ and their ratios to $\mathrm{C}$ and $\mathrm{O}$ (i.e. $\mathrm{B} / \mathrm{C}, \mathrm{B} / \mathrm{O}$, $\mathrm{Be} / \mathrm{C}, \mathrm{Be} / \mathrm{O}, \mathrm{Li} / \mathrm{C}$ and $\mathrm{Li} / \mathrm{O}$ ), predicted from the GALPROP and DRAGON2 cross sections parametrizations. These analyses are performed first for each ratio separately (what we call "Independent analysis") and, then, combining all of them ("Combined analysis"). The Combined analysis also includes nuisance parameters that allow the normalization of the cross sections parametrizations to be rescaled. Therefore, this analysis adds to the fit procedure a scale factor for each of the secondary CRs studied here $\left(\mathcal{S}_{B}, \mathcal{S}_{B e}\right.$ and $\left.\mathcal{S}_{L i}\right)$, since we are only able to evaluate our predictions against their total fluxes (i.e. the sum of fluxes of each isotope) - there is no experimental data on isotopic fluxes of these CRs above a few $\mathrm{GeV} / \mathrm{n}$. Here, we summarize the most remarkable results obtained from these analyses; the complete results can be found in the form of tables in the Appendix of Ref. [5].

\subsubsection{Main findings from the independent analyses}

Firstly, it is important to remark that each of the ratios (also in the combined analysis and with both cross sections) favors a negative value of $\eta$, which means that there is a change in the trend of the diffusion coefficient at energies below a few $\mathrm{GeV} / \mathrm{n}$. This involves that $\mathrm{CR}$ particles propagate more effectively, which can be theoretically motivated by dissipation of plasma waves [15]. 
Then, we observe a large dispersion in the determination of the normalization of the diffusion coefficient, $D_{0}$, which is expected given the uncertainties in the normalization of the cross sections parametrizations, discussed above. The predictions for the Li ratios from both parametrizations show huge differences, while those from the $\mathrm{B}$ ratios seem to be compatible within $1 \sigma$, as expected. However, the other parameters, related with the energy dependence of the predicted spectra, also show certain differences, although generally within $3 \sigma$. Interestingly, the value obtained for $\delta$ for the $\mathrm{B}$ and $\mathrm{Be}$ ratios is around $0.40-0.45$, for the GALPROP cross sections and $0.43-0.45$ for the DRAGON2 ones, while a value around $0.39-0.41$ is found for the Li ratios. Although the $1 \sigma$ statistical uncertainty in the determination of this value is around \pm 0.01 in this analysis, the uncertainties related to the spallation cross sections for Li production are specially large, so that this difference is not high enough to claim that the Li spectrum is harder than the spectra of the other species. The largest difference comparing the predictions from both cross sections parametrizations $(>2 \sigma)$ is found for the comparison between the Be ratios of both cross sections.

The values of $\eta$ and $V_{A}$ found are statistically compatible (at least within $2 \sigma$ ) between both cross sections, which make us conclude that these parametrizations only lead to slight differences in the prediction of these propagation parameters from the independent fits of the ratios of $\mathrm{B}, \mathrm{Be}$ and Li to $\mathrm{C}$ and $\mathrm{O}$. Nevertheless, it must be remarked that a larger dispersion of the predicted parameters (mainly for $D_{0}$ and $\delta$ ) is found for the $\mathrm{B}, \mathrm{Be}$ and Li rations with the GALPROP cross sections.

\subsubsection{Main findings from the combined analyses}

Once we combine all the ratios and introduce the scaling factors able to modify the normalization of the cross sections, we observe that the predicted values of $\eta(\sim-0.75)$ and $D_{0}$ $\left(\sim 1.12 \times 10^{28}\left[\mathrm{~cm}^{2} / \mathrm{s} / \mathrm{kpc}\right] \times H[\mathrm{kpc}]\right)$ are well compatible within the $1 \sigma$ statistical uncertainties. The value of the effective Alfén velocity, $V_{A}$, seems to be different between both parametrizations by slightly above $2 \sigma$ (although it remains around $30 \mathrm{~km} / \mathrm{s}$ ), which is due to the fact that both parametrizations implement a slightly different energy dependence for different interaction channels. Then, the value of the spectral index of the diffusion coefficient, $\delta$, is 0.42 and 0.40 , for the DRAGON2 and GALPROP parametrizations, respectively, with a $1 \sigma$ error of about \pm 0.01 . Nevertheless, despite the fact that these parameters are not very different between both cross sections, we
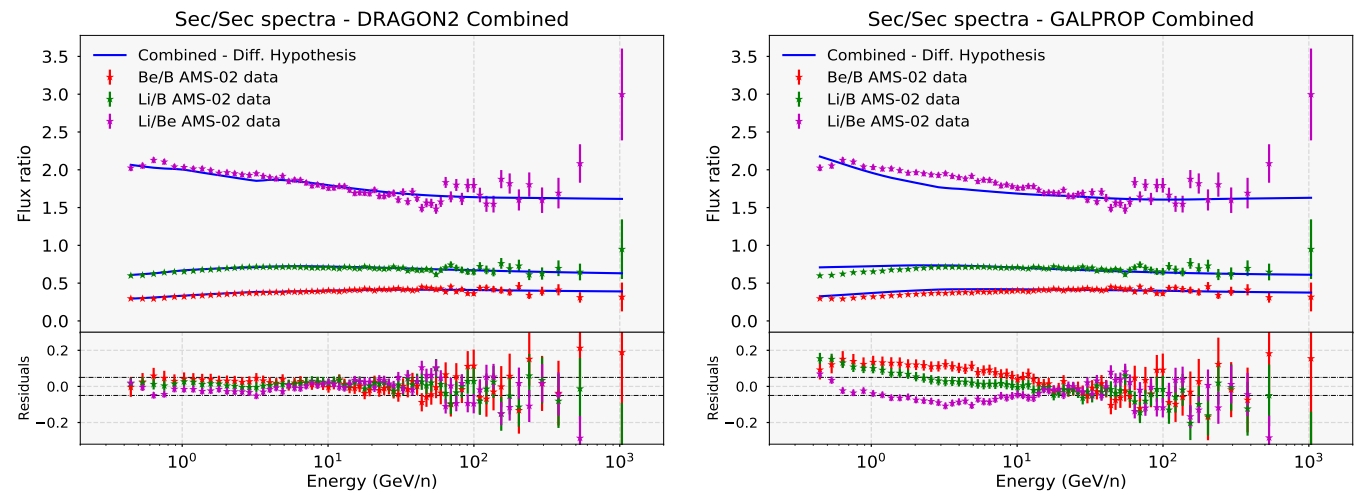

Figure 4: Flux ratios among B, Be and Li obtained from the combined analysis for the DRAGON2 (left) and GALPROP (right) cross sections, compared to the AMS-02 experimental data. 
find that the predicted best-fit parameters lead to a very good agreement to data for the DRAGON2 parametrizations while a considerably poorer agreement for the GALPROP ones, as shown in fig. 4. Finally, the values of the scale factors are $\mathcal{S}_{B}=0.99, \mathcal{S}_{B e}=0.9, \mathcal{S}_{L i}=1.26$ for the GALPROP cross sections and $\mathcal{S}_{B}=1.05, \mathcal{S}_{B e}=0.99, \mathcal{S}_{L i}=0.97$ using DRAGON2.

\section{Conclusions}

In this work, we have presented a summary of various recent studies on cross sections uncertainties and their impact on our CR predictions. We have discussed that the main source of uncertainties is the normalization of the cross sections parametrizations and how this affects our conclusion on the nature of the secondary CRs and their production. Then, we have revised the propagation parameters predicted from the DRAGON2 and GALPROP parametrizations and demonstrated that the spectra of the main secondary CR species can be simultaneously reproduced including just a renormalization factor with the DRAGON2 parametrizations. We conclude here that, due to the importance of spallation cross sections measurements in our codes of CR propagation, new measurements above a few $\mathrm{GeV} / \mathrm{n}$ are crucial to update the current parametrizations and that these parametrizations can be further refined combining different $\mathrm{CR}$ observables. The future measurements of isotopic fluxes will provide an important boost in this respect.

\section{References}

[1] V. Berezinskii et al., Astrophysics of cosmic rays, Vol. 393 (North-Holland Amsterdam, 1990).

[2] L. Derome et al., Astronomy \& Astrophysics 627, A158 (2019).

[3] Y. Genolini et al., Phys. Rev. C98, 034611 (2018), arXiv:1803.04686 [astro-ph.HE] .

[4] P. de la Torre Luque et al., JCAP 03, 099 (2021), arXiv:2101.01547 [astro-ph.HE] .

[5] P. De La Torre Luque et al., Accepted in JCAP (2021), arXiv:2102.13238 [astro-ph.HE] .

[6] P. de la Torre Luque, "tospines/Customised-DRAGON2_beta: First release of the customized DRAGON2_beta code," (2021).

[7] C. Evoli et al., JCAP 02, 015 (2017), arXiv:1607.07886 [astro-ph.HE] .

[8] C. Evoli et al., JCAP 07, 006 (2018), arXiv:1711.09616 [astro-ph.HE] .

[9] C. Evoli et al., Phys. Rev. D 99, 103023 (2019), arXiv:1904.10220 [astro-ph.HE] .

[10] M. Aguilar et al. (AMS Collaboration), Phys. Rev. Lett. 120, 021101 (2018).

[11] M. J. Boschini et al., The Astrophysical journal 889, 167 (2020), arXiv:1911.03108 .

[12] M. Aguilar et al. (AMS), Phys. Rev. Lett. 126, 081102 (2021).

[13] M. J. Boschini et al., (2021), arXiv:2106.01626 [astro-ph.HE] .

[14] M. Aguilar et al. (AMS Collaboration), Phys. Rev. Lett. 124, 211102 (2020).

[15] V. S. Ptuskin et al., The Astrophysical Journal 642, 902 (2006). 